\title{
Toxins in the food chain, and why quality supplementation is necessary
}

Volume 7 Issue 5 - 2017

\author{
Eldon Dahl \\ Founder and CEO of Life Choice TM nutraceutical product line, \\ USA
}

Correspondence: Eldon Dahl, Eldon Dahl, Doctorate in natural medicine, Founder and CEO of Life Choice Ltd, USA, Email lifechoicedoc@telus.net

Received: June 09, 2017| Published: June 20, 2017
The series of questions that individuals ask more often is, "Are supplements necessary? Do we not get all of the necessary nutrients, vitamins and minerals from the food that we eat? And if we eat a regular, balanced diet, is that not enough to keep our bodies healthy and fit?"

If you were to ask those questions prior to the turn of the 20th century, or even into the first quarter of the 20th century, the answer would have been yes. But today, given the fact that we do not have pure food anymore, the answer is no!

Today, our oceans are in terrible shape. The fish that we catch have high levels of poisons and toxins. The Environmental Protection Agency and Food and Drug Administration have released minimum weekly levels for fish consumption. Women who are pregnant, breastfeeding, or trying to become pregnant are recommended to eat up to 12 ounces of fish per week. The major problem with this advice is that mercury is abundant in many types of fish, and no level has been found safe, so these toxins will to be transferred from mother to fetus.

On the land, farmers use GMO seeds, and cross contamination can go on for miles.

Did you know that over $80 \%$ of US corn and cotton crops and $94 \%$ of soy crops are genetically modified? GM sugar beets were introduced in 2008, and within the first year, $90 \%$ of the sugar beets grown in the U.S. were genetically modified (GMO)--that is $80 \%$ of the world's supply.

In the soil, a study published in the scientific journal Entropy has revealed the long-term effects of glyphosate, which is most widely utilized in Roundup ${ }^{\circledR}$ herbicide. The study found that Roundup could predispose humans to conditions such as obesity, Alzheimer's, Parkinson's, etc.

In plants, Roundup interferes with a biochemical pathway called the shikimate pathway. While this pathway is not found in humans, it is found in bacteria, such as the bacteria found in a human GI tract. In the bacteria, amino acids such as tyrosine, tryptophan, and phenylalanine are depleted, which, in humans, can contribute to the diseases mentioned above.

Chemical fertilizers, herbicides and pesticides are used on a regular basis. These chemicals go into the root of the plant and eventually become part of the plant themselves. This is very concerning when we talk about rice, corn, soybeans and other food staples that are consumed around the world in large quantities.

In chickens, another study found that glyphosate could predispose chickens to carry higher levels of Salmonella or other harmful bacteria. In fact, the beneficial GI bacteria were susceptible to glyphosate, while the harmful bacteria were resistant to it.
Chickens, cattle, and pigs from birth are injected with steroids and antibiotics to allow for quicker growth, so they can go to market earlier.

These chemicals do not break down, and end up entering our bodies, only to accumulate as toxins within our own system. This accumulation of antibiotics leaves us resistant to antibiotics and susceptible to a weakened immune system to the point that common bacteria could become fatal. This overload of antibiotics leaves medication ineffective, and pharmaceutical companies have not introduced a completely novel antibiotic since 1987. Because of this, we're losing ground and not keeping pace with superbugs' ability to develop resistance to the current antibiotics.

Interestingly, this is why so many people today think they are allergic to gluten, and why Crohn's and Celiac disease are so abundant. People are not allergic to gluten; they are allergic to toxins - we do not have pure food any longer.

As the selection of non-GMO products decreases, it is all the more important that we take the power into our own hands in order to build a healthy immune system to ward off the detrimental effects. Globally we are fighting for our health, and that is why educated choices must be made in the food we eat, and the therapeutic quality of the supplements we take. The lifestyle we plan on leading must be approached proactively; this is not only necessary, but critical in order to having healthy life.

\section{Acknowledgments}

None.

\section{Conflicts of interest}

Author declares there are no conflicts of interest.

\section{Funding}

None. 